\title{
39 Geographic Information Science: The Grand Challenges
}

\section{MICHAEL F. GOODCHILD}

Many chapters in this book have touched on aspects of the research agenda - the research questions that arise in the mind of a user of GIS; the research problems that need to be solved to enable the next generation of GIS technology; in essence the science behind the systems. Over the past twelve years there have been various broader efforts to define a more-or-less-complete agenda for GI Science, and many of them have been framed in terms of challenges to the research community. More broadly still, many fields of science have attempted to provide long-term motivation - and not incidentally to open sources of funding - by identifying grand challenges, themes that are capable of directing research to a common, distant, but imaginable end. President John F. Kennedy's famous challenge of 1960 - to "put a man on the Moon by the end of this decade" - resulted in an unprecedented peacetime integration of science, engineering, political support and of course, a successful ending. In a somewhat similar vein biologists have been calling for the completion of "the web of life", the identification of all of the species (only a fraction of which are currently known to science; May 1988). The mapping of the human genome is a similarly integrated effort across a multitude of laboratories and institutions, tied together by computer networks. Could there be a grand challenge in GI Science that could drive a decade-long effort by the research community?

In the first part of the chapter I review the various published research agendas of GI Science. I then discuss a further type of challenge: the need to understand the nature of the 
geographic world. The third major section examines broader themes within science, and the degree to which they might translate into research challenges for GI Science and institutional challenges for the GI Science community. The final major section discusses the concept of Digital Earth as a grand challenge for GI Science.

\section{The Research Agendas of GI Science}

\subsection{The late 1980s}

It seems appropriate to begin this review in the late 1980s, because several events at that time helped to dramatically alter the landscape of the mapping sciences. In the UK, the Department of the Environment's Committee of Enquiry into the Handling of Geographic Information (the Chorley Committee; Department of the Environment 1987) saw three specific stimuli: the rapidly falling costs of hardware, which had reduced the cost of entry into GIS and related activities from $\$ 500,000$ at the beginning of the decade to $\$ 10,000$ at the end; the advent of COTS (commercial, off-the-shelf) software to perform the basic operations of GIS; and rapid growth in the availability of spatially referenced digital data. In the U.S., the National Science Foundation announced a competition for a National Center for Geographic Information and Analysis (NCGIA), to advance the theory and methods of GIS, to promote the use of GIS across the sciences, and to increase the nation's supply of experts in GIS.

Rhind (1988) presented a research agenda for GIS, identifying problems in what he termed the handling of geographic data: the volumes of data involved, the numerous types of queries that might be addressed, the prevalence of uncertainty in geographic data; the need for integration of data among organizations; and the lack of awareness of such issues as 
scale. He recognized that the solution of the more generic of these issues would come with time from mainstream information technology; but that issues that were more specific to the geographic case would have to be solved by an active research community focused on GIS. He saw a substantial role for knowledge-based or expert systems in the automated extraction of features from images, the integration of disparate data sets, the development of intelligent search procedures, the automation of cartographic generalization, the development of machine-based tutors, and the elicitation of knowledge from data. He also recognized the importance of research into better methods of visualization for geospatial data, the role of organizations, the legal issues of liability and intellectual property, and the costs and benefits of GIS.

The NCGIA research agenda (National Center for Geographic Information and Analysis 1989) has much in common with Rhind's, but already shows signs of a search for the more fundamental issues of GI Science, in contrast to the practical issues of GIS. Five major research areas are identified:

- Spatial analysis and spatial statistics, the techniques used to model uncertainty in geospatial data, to mine data for patterns and anomalies, and to test theories by comparison with reality.

- Spatial relationships and database structures, addressing the representation of real geographic phenomena in digital form, and the interface between digital structures and human reasoning.

- Artificial intelligence and expert systems, reflecting Rhind's concern for the role of advanced machine intelligence in GIS operations. 
- Visualization, and the need to advance traditional cartography to reflect the vastly greater potential of digital systems for display of geographic data.

- Social, economic and institutional issues, the host of social issues surrounding GIS.

The NCGIA went on to propose 12 specific research initiatives within this general framework:

- Accuracy of spatial databases, focusing on error models for geographic data with strong links to the discipline of statistics.

- Languages of spatial relations, including principles of spatial cognition and linguistics.

- Multiple representations, the need to integrate representations of the Earth's surface at different scales and levels of generalization.

- Use and value of geographic information in decision making.

- Architecture of very large GIS databases.

- Spatial decision support systems, the design of systems to support decisionmaking by groups of stakeholders.

- Visualization of the quality of geographic information, through methods that explicitly display information about the uncertainty associated with data.

- Expert systems for cartographic design, using intelligent systems to augment the skill of cartographers.

- Institutions sharing geographic information, including research on the impediments to sharing between agencies. 
- Temporal relations in GIS, the extension of GIS data models to include time.

- Space-time statistical models in GIS, the extension of spatial analysis to include time.

- Remote sensing and GIS, researching the issues involved in the integration of data acquired by remote sensing with data from other sources.

Eventually, NCGIA sponsored a total of 21 research initiatives between 1988 and 1996; reports, papers, and other products are available at http://www.ncgia.org.

\subsection{The 1990s}

Very substantial progress was made on most of these topics in the years following their publication. In addition, four factors contributed to the evolution of these research agendas in the 1990s: (1) the continued arrival of new technologies, including most notably the WWW, the Global Positioning System, object-orientation, and mobile computing; (2) the broadening of the research community, to include active participation by new disciplines, notably cognitive science, computer science, and statistics; (3) the trend away from technical issues of systems to fundamental issues of science; and (4) the recognition that certain topics were in effect dead ends. This last factor perhaps accounts for the virtual disappearance of expert systems, despite their prominence in Rhind's 1988 agenda.

In 1996 the recently formed University Consortium for Geographic Information Science (http://www.ucgis.org) published the first edition of its research agenda (University Consortium for Geographic Information Science 1996), the result of a successful consensusbuilding exercise amongst the thirty or so research institutions that were then members (the number has since risen to more than 60 ). The agenda had ten topics: 
- Spatial data acquisition and integration, including new sources of remote sensing, ground-based sensor networks, and fusion and conflation of data from different sources.

- Distributed computing, and the issues of integrating data and software over large heterogeneous networks.

- Extensions to geographic representations, addressing particularly the third spatial dimension and time.

- Cognition of geographic information, including studies of the processes by which people learn and reason with geographic data, and interact with GIS.

- Interoperability of geographic information, including research to overcome the difficulties of different formats and lack of shared understanding of meaning.

- Scale, and the complex issues surrounding representations at different levels of detail.

- Spatial analysis in a GIS environment, advancing the analytic capabilities of GIS.

- The future of the spatial information infrastructure and the institutional arrangements that provide the context for GIS.

- Uncertainty in geographic data and GIS-based analysis, including the modeling and visualization of data quality.

- GIS and society, the study of the impacts of GIS on society, and the societal context in which the technology is used.

UCGIS later added four emerging themes to the list: 
- Geospatial data mining and knowledge discovery, the development of methods for extracting patterns and knowledge from very large data sources.

- Ontological foundations of geographic information science, addressing the fundamental components on which our knowledge of the Earth's surface is based.

- Geographic visualization.

- Remotely acquired data and information in GI Science.

The UCGIS completely revised this list in 2002, replacing it with lists of long-term research challenges and short-term research priorities.

The theme of visualization has been taken up and developed by the International Cartographic Association's (ICA's) Commission on Visualization and Virtual Environments. The four-part research agenda (MacEachren and Kraak 2001) includes:

- Cognitive and usability issues in geovisualization.

- Representation and its relationship with cartographic visualization.

- The integration of geographic visualization with knowledge discovery in databases and geocomputation.

- User interface issues for spatial information visualization.

More generally, the list of commissions of the ICA reflects the broad interests of the association, including many research topics.

The International Society for Photogrammetry and Remote Sensing also provides a useful insight into contemporary research needs, with particular emphasis on imaging systems. Its seven permanent commissions address:

- Sensors, platforms, and imagery. 
- Systems for data processing, analysis, and representation.

- Theory and algorithms.

- Spatial information systems and digital mapping.

- Close-range vision techniques.

- Education and communications.

- Resource and environmental monitoring.

In 1998, NSF sponsored a workshop under its Digital Government Initiative to explore ways of improving geographic information services (National Computational Science Alliance 1999). The workshop made ten recommendations, all aimed at advancing GIScience research:

- Advance research efforts directed toward the study of optimizing geographic query mechanisms and incorporating geometry and spatial relational operations.

- Develop improved mechanisms for storing and representing time-varying geospatial data.

- Support research on integrating spatial data fusion from multiple agencies, distributed data, and multiple collection devices.

- Support research on multiple representations/interfaces focused on task-specific (procedural) workflow classes.

- Support research in developing algorithms for knowledge discovery applied to very large, frequently updated spatial datasets such as those derived from space-borne Earth-monitoring sensors. 
- Support research in the theory and methods of representing data with varying degrees of exactness and reliability.

- Support research in the context of decision-making to improve the representation of diverse data and the dynamics of geographic phenomena.

- Extend the promise of cognitive research to make geographic information technologies more accessible to inexperienced and disadvantaged users and also examine how government information policies affect access to and use of geospatial data for a broad spectrum of public and private sector stakeholders.

- Support research to examine commerce's issues in geospatial information such as preserving privacy despite geographic locators and breaking potential bottlenecks in distributing geographic information services due to GIS's unique workflow processes.

- Develop a Geospatial Digital Government Prototyping Center to create a network for testing and developing processes consistent with US priorities for geographic information technologies and services in the government workplace.

More recently, the Center for Mapping of The Ohio State University organized a 2002 workshop on Geographic Information Science and Technology (GIS\&'T) in a Changing Society. The perspective of the workshop was notably toward societal issues, and it identified six research areas:

- Geospatial data availability: its sources and influences.

- GI S\&T workforce studies.

- Conditions associated with the adoption of GI S\&T-based approaches. 
- Spatial understandings, or the cognitive ability of people to work with spatial data.

- Cross and longitudinal studies of the use of GI S\&T.

- Improved tools for the societal evaluation of GI S\&T activities.

Finally, the National Research Council's Computer Science and Telecommunications Board reported in 2003 on a study of research needs at the intersection of GI Science and computer science (National Research Council 2003). It identified two over-arching themes: the need for an integrative, multidisciplinary approach to research, and the need to address issues of policy. Within this context, it proposed eight research topics:

- Accessible location-sensing infrastructure, based on systems that know their location.

- Mobile environments, freeing users from the desktop.

- Geospatial data models and algorithms.

- Geospatial ontologies.

- Geospatial data mining.

- Geospatial interaction technologies.

- Geospatial for everyone, everywhere.

- Collaborative interactions with geoinformation.

\section{Towards Synthesis}

Although each of these studies and reports contains an extensive list of topics, several common themes are apparent, as well as several consistent trends through time. Mark (2003) has analyzed several of the lists, including the topics included in my 1992 paper in which I proposed the term geographic information science (Goodchild 1992). 
A somewhat different approach to framing the research agenda was taken by NCGIA's Project Varenius, a research effort begun in 1996 to advance the fundamentals of geographic information science, with funding from the National Science Foundation (Goodchild et al. 1999, Mark et al. 1999, Egenhofer et al. 1999, Sheppard et al. 1999). In this strikingly simple model, GI Science was anchored by three concepts - the individual, the computer, and society - represented by a triangle, with GI Science at the core. Research about the individual would be dominated by cognitive science, and its concern for understanding of spatial concepts, learning and reasoning about geographic data, and interaction with the computer. Research about the computer would be dominated by issues of representation, the adaptation of new technologies, computation, and visualization. Finally, research about society would address issues of impacts and societal context. Many research issues would involve the interaction between the three corners of the triangle.

\section{A Natural Science?}

It will be clear from the previous section that the research agenda of GI Science is diverse, covering issues of technology, society, and human cognition. This section introduces a fourth, which as been largely neglected to date - the dependence of GI Science on an understanding of the nature of the Earth's surface.

Many decisions are made in the design of a GIS, and more generally the design of any technology that must process geographic information. They include decisions about data models, data structures, indexing schemes, and algorithms - and about the set of analytic routines and processing functions. Many of these decisions are in turn dependent on expectations about the nature of geographic information. For example, a decision to 
represent rivers as polylines (sequences of points connected by straight segments) is a compromise, balancing the disadvantages of representing a smoothly bending river with a series of straight lines and sharp corners, against the advantages of using such a simple geometry (intersections between straight lines are much easier to compute than intersections between curves). Yet while there clearly are expectations about the nature of geographic information, very few attempts have been made to research the topic systematically, or to assemble what is known in coherent fashion.

The best-known statement is probably Tobler's, generally known as the First Law of Geography: "all things are related, but nearby things are more related than distant things". This first appeared in a paper on urban growth in Detroit (Tobler 1970), and formed the subject of a recent forum in the Annals of the Association of American Geographers (see Sui 2004, Barnes 2004, Miller 2004, Phillips 2004, Smith 2004, Goodchild 2004, and Tobler 2004 for additional details). More formally it is a statement about the endemic presence of positive spatial autocorrelation in geographic information, and thus of the principle underlying the entire field of geostatistics.

The consequences of Tobler's First Law (TFL) for GIS design are profound. If it were not true, and nearby things were as different as distant things, then all forms of spatial interpolation would be impossible, along with the derivative processes of contour mapping and resampling. All advanced GIS data structures would be impossible, since there would be no basis for assuming that terrain could be represented as a mesh of triangles, or that points with similar characteristics could be grouped into polygons. One can go further and argue that a geographic world without TFL would be impossible to learn about or describe, since every point would be independent of its most immediate surroundings. There are of course 
exceptions, and TFL is not a deterministic law. It is possible for example for spatial independence to exist over distances in excess of what geostatisticians would define as the phenomenon's range, and it is possible for negative spatial autocorrelation to exist at certain scales (at the scale of the cells in a checkerboard, for example).

Anselin (1989) has argued that TFL, or the principle of positive spatial autocorrelation, is one of two endemic properties of geographic information. The other is spatial heterogeneity, or the tendency for properties to vary from one area to another over the Earth's surface. In the terms of spatial statistics, this is a first-order effect, or a property of places taken one at a time, while TFL describes a second-order effect, a property of places taken two at a time. For that reason it might be preferable if TFL were the Second Law, and spatial heterogeneity the basis of a First Law.

The consequences of spatial heterogeneity are also profound. If the Earth's surface is heterogeneous, it follows that standards and design decisions adopted in one region, and designed for the conditions of that region, will be different from those adopted in other regions. Spatial heterogeneity thus explains the lack of interoperability between the various classification schemes used in geographic information, and the tension between local geodetic datums and global ones. It dictates that the results of any analysis will depend explicitly on the bounds of the analysis, and will change when the bounds change. It also makes a compelling case for the new place-based or locally centered methods of spatial analysis, such as Geographically Weighted Regression (Fotheringham et al. 2002) and LISA (Anselin 1995).

Additional generalizations can be made not so much about the nature of geographic information, as about the nature of its representation. Many geographic phenomena reveal 
more detail as they are examined more closely, and the rate at which additional detail is revealed is to some degree predictable. Mandelbrot (1982) termed such phenomena fractals, and showed that fractal properties were broadly characteristic of geographic information. They imply a degree of predictability in the effects of scale change, allowing better-informed design decisions to be made regarding hierarchical structures in GIS. I have argued that the endemic presence of uncertainty in GIS representations has profound impacts on GIS design, and leads to an entirely different approach to the representation of position (Goodchild 2002).

These cases all point to the need for GI Science to address the nature of geographic information, and in effect to become in part a natural science, comparable to physics, chemistry or biology, with its own unique domain of study in the natural world. Of course this vision of the domain of GI Science must include phenomena that are of human origin, or are influenced by humans.

\section{Broader Themes}

Since its inception in the 1960s, GIS has become useful and almost indispensable in a vast range of human activities, from Earth science to human health, and from transportation to resource management. It is the enabling technology that has permitted utility companies to move to a higher level of efficiency in their management of distributed networks, and package delivery companies to save millions in delivery costs. Advances in GI Science are essential to the further development of GIS, and the key to the success of the technology's next generation. 
Despite its importance, however, GIS remains a comparatively small application of information technology, and as such it must rely on the larger mainstream for many developments. The relational and object-oriented databases now widely used in GIS are mainstream products, that would probably not have been developed if GIS was their only market; and at the same time GIS has relatively little influence on such developments.

In this context, one might argue that the future of GIS lies not in the specialized research agenda of GI Science, but in broader research agendas that will determine the future of information technology. Many of the research agenda topics identified above are indeed more general than GIS, and one can expect a broader set of minds to be interested in them. To what extent, for example, are issues of ontology and interoperability unique to GIS, and to what extent are they common to a much larger domain? Semantic interoperability is a problem common to all applications that rely on the meaning of terms, and one might therefore expect solutions to come from a number of disciplines, not only from GI Science.

This argument has two important implications. First, it suggests that GI Science must continue to look more broadly to developments that may have potential for GIS. A case in point is the Grid, a term that encompasses a range of technologies and research efforts aimed at integrating the distributed computing resources of widely dispersed communities into transparent wholes. The bandwidth of the Internet now makes it possible for the components of computing - the processor, data, and software - to be distributed virtually anywhere, and for a user at a desktop to access what amounts to a previously unimaginable resource. Services need no longer be provided at the desktop, but can be invoked from servers located anywhere on the network, and might include any of the functions currently 
performed locally by a GIS. Several GI services are already available (the Geography Network, http://www.geographynetwork.com, includes a directory), but much research remains to be done to explore GIS applications of the Grid.

Second, it suggests that it is in the strategic interest of the GI Science community to generalize its efforts wherever possible. One such generalization would be from geographic to spatial, to explore domains defined by spaces other than that of the Earth's surface. The space of the human brain, for example, has similarities and also important differences, that can lead both to new applications for GIS, and also to the cross-fertilization of research. While there is only one Earth, each human brain is different, requiring brain researchers to develop techniques for defining a generic brain, and for mapping each individual brain to it. One can ask whether other spaces display the same properties as are observed for geographic space, such as TFL, and whether information system design considerations are therefore the same or different for these other spaces.

\section{A Grand Challenge: Digital Earth}

The previously cited NSF workshop (National Computational Science Alliance 1999) asked whether grand challenges existed for GIS, and in its report listed four research themes that it felt might merit this distinction:

- To find ways to express the infinite complexity of the geographical world in the binary alphabet and limited capacity of a digital computer (the representation challenge);

- To find ways of summarizing, modeling, and visualizing the differences between a digital representation and real phenomena (the uncertainty challenge); 
- To achieve better transitions between cognitive and computational representations and manipulations of geographic information (the user interface challenge); and

- To create simulations of geographic phenomena in a digital computer that are indistinguishable from their real counterparts (the modeling challenge; in effect a Turing test of GIS-based modeling).

All of these have intellectual depth, but somehow lack the compelling appeal of a megaproject. But the concept of Digital Earth (DE) perhaps has the ability to capture popular imagination. The term was coined by Gore (1992) and elaborated in a much-quoted 1998 speech (http:/ / digitalearth.gsfc.nasa.gov/VP19980131.html):

"Imagine, for example, a young child going to a Digital Earth exhibit at a local museum. After donning a head-mounted display, she sees Earth as it appears from space. Using a data glove, she zooms in, using higher and higher levels of resolution, to see continents, then regions, countries, cities, and finally individual houses, trees, and other natural and man-made objects. Having found an area of the planet she is interested in exploring, she takes the equivalent of a 'magic carpet ride' through a 3-D visualization of the terrain. Of course, terrain is only one of the numerous kinds of data with which she can interact. Using the system's voice recognition capabilities, she is able to request information on land cover, distribution of plant and animal species, real-time weather, roads, political boundaries, and population. She can also visualize the environmental information that she and other students all over the world have collected as part of the GLOBE project. This information can be seamlessly fused with the digital map or terrain data. She 
can get more information on many of the objects she sees by using her data glove to click on a hyperlink. To prepare for her family's vacation to Yellowstone National Park, for example, she plans the perfect hike to the geysers, bison, and bighorn sheep that she has just read about. In fact, she can follow the trail visually from start to finish before she ever leaves the museum in her hometown. She is not limited to moving through space, but can also travel through time. After taking a virtual field-trip to Paris to visit the Louvre, she moves backward in time to learn about French history, perusing digitized maps overlaid on the surface of the Digital Earth, newsreel footage, oral history, newspapers and other primary sources. She sends some of this information to her personal e-mail address to study later. The timeline, which stretches off in the distance, can be set for days, years, centuries, or even geological epochs, for those occasions when she wants to learn more about dinosaurs."

This vision of DE raises numerous problems (Goodchild 1999, 2000). First, it implies that data structures can be found that support a smooth zooming from resolutions as coarse as $10 \mathrm{~km}$ (whole-Earth view) to near $1 \mathrm{~m}$ (individual houses and trees). Research on this topic has been under way for many years, and implementations are now widely available (in ESRI's ArcGlobe and Google Earth, for example). Second, it presents enormous problems of data volume, since there are $5 \times 10^{14} \mathrm{~m}^{2}$ on the Earth's surface. It raises problems of visual rendering, since although some data (e.g. terrain elevation) are easily rendered in threedimensional views, others (e.g. average income) would have to be communicated symbolically. Perhaps more problematic are the institutional issues, since DE would require 
smooth interoperability between data sets, and collaboration by numerous data suppliers and custodians. But all of these are of course exactly what is required for a grand challenge - the collaboration of many disciplines, agencies, and communities in progress towards a commonly held vision.

$\mathrm{DE}$ is presented by Gore as an educational tool, a way for a younger generation to acquire knowledge of the planet, and particularly of its environmental problems and ways in which they might be solved. Another major benefit of DE would lie in its ability to serve as an experimental environment, allowing planners to evaluate the consequences of management and development alternatives. One can imagine evaluating the consequences of a steady rise in atmospheric $\mathrm{CO}_{2}$ using $\mathrm{DE}$, as an alternative to the infinitely costly and dangerous experiment that humanity is currently conducting on the real thing (for a Japanese effort along these lines see http://www.nec.com/global/features/index $9 \cdot$ html). GIS is currently used for this purpose, of course, but only over much smaller domains. Again, these are the kinds of massive benefits one would expect from the solution of a grand challenge.

\section{Conclusions}

In the previous sections I have implied numerous criteria for grand challenges, some explicitly and some implicitly. In this final section I will review these, and examine the extent to which they are satisfied by the various proposals.

First, a grand challenge should focus many apparently disparate forms of research on a common goal. DE clearly satisfies this test, since it involves researchable issues that are both technical and institutional, and touch on the disciplines of geography, computer science, 
cognitive science, and any of the many disciplines that study the processes responsible for the evolution of the Earth's surface.

Second, there are expectations regarding the magnitude of a grand challenge, whether measured in numbers of investigators, levels of funding, or numbers of papers produced. DE has already spawned several conferences, and research on many of its sub-problems continues throughout the GI Science community, though often without explicit recognition of its relevance to DE. DE has many pseudonyms: Virtual Earth, Earth System, and Digital Globe all produce numerous WWW hits on DE-like projects. The Japanese Earth Simulator project alone represents an investment of several hundred million dollars.

Third, a grand challenge should capture the popular imagination, and thus political support. The results to date on this test are much less clear for DE, and also for GIS and GI Science. While there is now very extensive name recognition of GIS, and its value is without question, its development clearly has not attracted the kind of widespread public attention accorded to the lunar landings, or even the mapping of the human genome. It is, quite simply, a very useful tool that we can no longer do without - and DE a very useful vision of where it might be headed in the years to come. 


\section{References}

Anselin L 1989 What Is Special about Spatial Data? Alternative Perspectives on Spatial Data Analysis. Santa Barbara, CA, National Center for Geographic Information and Analysis Technical Paper No 89-4

Anselin L 1995 Local indicators of spatial association: LISA. Geographical Analysis 27: 93-115

Barnes T J 2004 A paper related to everything but more related to local things. Annals of the Association of American Geographers 94: 278-83

Department of the Environment 1987 Handling Geographic Information: The Report of the Committee of Enquiry Chaired by Lord Chorley. London, Her Majesty's Stationery Office

Egenhofer M J, Glasgow J, Günther O, Herring J R, and Peuquet D J 1999 Progress in computational models for representing geographic concepts. International Journal of Geographical Information Science 13: 775-98

Fotheringham A S, Brunsdon C, and Charlton M 2002 Geographically Weighted Regression: The Analysis of Spatially Varying Relationships. New York, John Wiley and Sons

Goodchild M F 1992 Geographical information science. International Journal of Geographical Information Systems 6: 31-45

Goodchild M F 1999 Implementing Digital Earth: A research agenda. In Towards Digital Earth: Proceedings of the International Symposium on Digital Earth, Beijing, China

Goodchild M F 2000 Cartographic futures on a digital Earth. Cartographic Perspectives 36: 3-11

Goodchild M F 2002 Measurement-based GIS. In Shi W, Fisher P F, and Goodchild M F (eds) Spatial Data Quality. New York, Taylor and Francis: 5-17

Goodchild M F 2004 The validity and usefulness of laws in geographic information science and geography. Annals of the Association of American Geographers 94: 300-3 
Goodchild M F, Egenhofer M J, Kemp K K, Mark D M, and Sheppard E S 1999 Introduction to the Varenius project. International Journal of Geographical Information Science 13: $731-46$

Gore A 1992 Earth in the Balance: Ecology and the Human Spirit. Boston, MA, Houghton Mifflin MacEachren A M and Kraak M J 2001 Research challenges in geo-visualization. Cartography and Geographic Information Science 18: 3-12

Mandelbrot B B 1982 The Fractal Geometry of Nature. San Francisco, CA, Freeman

Mark D M, Freksa C, Hirtle S C, Lloyd R, and Tversky B 1999 Cognitive models of geographical space. International Journal of Geographical Information Science 13: 747-74

Mark D M 2003 Geographic information science: Defining the field. In Duckham M, Goodchild M F, and Worboys M F (eds) Foundations of Geographic Information Science. New York, Taylor and Francis: 3-18

May R M 1988 How many species are there on earth? Science 247: 1441-9

Miller H J 2004 Tobler's First Law and spatial analysis. Annals of the Association of American Geographers 94: 284-9

National Center for Geographic Information and Analysis 1989 The research plan of the National Center for Geographic Information and Analysis. International Journal of Geographical Information Systems 3: 117-36

National Computational Science Alliance 1999 Toward Improved Geographic Information Services within a Digital Government: Report of the NSF Digital Government Initiative Geographic Information Systems Workshop. Champaign, IL, University of Illinois at Urbana-Champaign

National Research Council 2003 IT Roadmap to a Geospatial Future. Washington, D.C., National Academy Press 
Phillips J D 2004 Doing justice to the law. Annals of the Association of American Geographers 94: $290-3$

Rhind D W 1988 A GIS research agenda. International Journal of Geographical Information Systems 2: $23-8$

Sheppard E S, Couclelis H, Graham S, Harrington J W, and Onsrud H 1999 Geographies of the information society. International Journal of Geographical Information Science 13: 797-824

Smith J M 2004 Unlawful relations and verbal inflation. Annals of the Association of American Geographers 94: 294-9

Sui D Z 2004 Tobler's First Law of Geography: A big idea for a small world? Annals of the Association of American Geographers 94: 269-77

Tobler W R 1970 A computer movie: Simulation of population change in the Detroit region. Economic Geography 46: 234-40

Tobler W R 2004 On the First Law of Geography: A reply. Annals of the Association of American Geographers 94: 304-10

University Consortium for Geographic Information Science 1996 Research priorities for geographic information science. Cartography and Geographic Information Science 23: 115-27 